\title{
EFECTO DE FACTORES GENÉTICOS Y AMBIENTALES SOBRE EL RECUENTO DE CÉLULAS SOMÁTICAS EN GANADO LECHERO DE COSTA RICA
}

\author{
María Gabriela Mora*, Bernardo Vargas ${ }^{1 / *}$, Juan José Romero*, Jorge Camacho* \\ Palabras clave: Recuento de células somáticas; heredabilidad; salud de la ubre; vacas lecheras. \\ Keywords: Somatic cell count; heritability; udder health; dairy cows.
}

Recibido: 08/03/16

RESUMEN

El estudio evaluó el efecto de factores genéticos y ambientales sobre el Score de Células Somáticas [SCS $=\log _{2}(\mathrm{RCS} / 100000)+3$ ] en ganado lechero de Costa Rica. Se analizaron 198685 registros diarios, provenientes de 43535 lactancias, 23749 vacas lecheras, 237 hatos y 3 grupos raciales. Se utilizó un modelo animal mixto para evaluar efectos fijos de zona, hato dentro de zona, raza, número de lactancia, mes/ año de registro, periodo de lactancia y la covariable de producción de leche diaria; también para efectos aleatorios de ambiente permanente, hatoxsemental y vaca. Se calcularon la heredabilidad $\left(\mathrm{h}^{2}\right)$ y repetibilidad (r) para el Recuento de Células Somáticas (SCS), así como los valores de cría (BLUP) y confiabilidad para los individuos en la población. El promedio de SCS fue 3,30 ( $\mathrm{DE}=1,87)$ todos los efectos fijos, significativos. Entre zonas, SCS varió desde 3,4 (bh-p) a 3,7 (bmh-p). Para hatos dentro de zona, el SCS osciló entre 1,6 y 5,5. El SCS aumentó desde 2,7 en primera lactancia hasta 4,0 para lactancias $\geq 6$. El SCS fue mayor en Enero $(3,6)$ y menor en Abril $(3,4)$. Se observó un patrón descendente no lineal en SCS desde el $2004(4,3)$ hasta el 2015 $(3,3)$ y un patrón no lineal de SCS a lo largo de la lactancia, que inició en 3,7 para disminuir hasta 3,1 al día 60 posparto, mientras que aumentó 3,9 al día 365. Se detectó una reducción de 0,051 en

1 Autor para correspondencia. Correo electrónico: bernardo.vargas.leitón@una.cr
Aceptado: 05/07/16

\section{ABSTRACT}

Effect of genetic and environmental factors on somatic cell count of dairy cattle from Costa Rica. The study evaluated the effect of genetic and environmental factors on the Score of Somatic Cell Count [SCS $=\log _{2}$ (SCC/100 000)+3] in Costa Rica dairy cattle. A total of 198685 daily records from 43535 lactations, 23749 cows, 237 herds and 3 breeds were analyzed. A mixed animal model was assessed with zone, herd within zone, breed, number of lactation, month/year or recording, lactation period and daily milk yield as fixed effects; and permanent environment, herd $\times$ sire and cow as random effects. Heritability $\left(h^{2}\right)$ and repeatability (r) indices for SCS were estimated. Breeding values and reliabilities were estimated for all individuals in the population. The average value of SCS was 3.30 ( $\mathrm{SD}=1.87)$. All fixed effects were significant. SCS among zones ranged from 3.4 (bh-p) to 3.7 (bmh-p). For herds within zone, SCS ranged from 1.6 to 5.5. SCS increased from 2.7 in first lactation to 4.0 for lactation $\geq 6$. SCS was higher in January (3.6) and lower in April (3.4). A consistent downward trend was observed in SCS from year 2004 (4.3) to 2015 (3.3). A nonlinear pattern was observed in SCS throughout lactation, starting at 3.7, with a marked decrease to a minimum of 3.1 around day 60 postpartum, and a progressive increase to a maximum of 3.9

\footnotetext{
* Universidad Nacional, Posgrado Regional en Ciencias Veterinarias Tropicales, Costa Rica.
} 
SCS por cada aumento de $1 \mathrm{~kg}$ en producción diaria de leche. Los valores de $h^{2}$ y $r$ fueron 0,08 ( $\mathrm{DE}=0,008)$ y $0,33(\mathrm{DE}=0,004)$, respectivamente. La confiabilidad de los BLUP en vacas y toros fue de $0,40(\mathrm{DE}=0,16)$ y $0,44(\mathrm{DE}=0,20)$, respectivamente. Estos resultados son útiles para un mejor control de SCS a nivel de hato, basado en el manejo preventivo de grupos más propensos, la optimización del descarte y la selección de sementales. around day 365 postpartum. A reduction of 0.051 in SCS was observed for each increase of $1 \mathrm{~kg}$ in daily milk production. Values of $h^{2}$ and $r$ for SCS were $0.08(\mathrm{SD}=0.008)$ and $0.33(\mathrm{SD}=0.004)$, respectively. Breeding values ranged from -1 to +1 . Reliabilities of breeding values for cows and bulls were $0.40(\mathrm{SD}=0.16)$ and $0.44(\mathrm{SD}=0.20)$, respectively. These results can be used to improve SCS at the herd level, based on prevention in groups under higher risk, optimization of cow culling strategies and improved sire selection.

\section{INTRODUCCIÓN}

La mastitis es uno de los problemas de salud más frecuentes en vacas lecheras, y es una de las principales razones para la eliminación permanente de vacas en los hatos y causa de grandes pérdidas económicas al sector lácteo (USDA 2007). La presencia de esta enfermedad no sólo afecta físicamente a las vacas, sino también la calidad de su leche. Una de las medidas que más se utiliza actualmente a nivel mundial para evaluar la calidad de la leche y la salud de la ubre es el recuento de células somáticas (RCS). Las células somáticas están conformadas en su mayoría por leucocitos, los cuáles intervienen a nivel de la ubre para combatir la infección (Sharma et al. 2011).

En leche proveniente de cuartos no infectados, un recuento celular somático normal se encuentra por debajo de las $200000{\mathrm{CS} . \mathrm{ml}^{-1}}^{-1}$ (Harmon 2001); sin embargo, en animales en su primera lactancia, este conteo puede ser menor a $100000 \mathrm{CS}^{-\mathrm{ml}^{-1}}$. Asimismo, cuando se obtiene

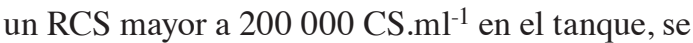
puede confirmar la presencia de mastitis subclínica en el hato lechero (García 2004).

Existen múltiples factores, tanto ambientales como genéticos, que pueden afectar el RCS.
Entre los factores ambientales, los procedimientos de ordeño, el espacio en el área de confinamiento o los materiales de las camas, son ejemplos de factores que inciden en los RCS (Lévesque 2004, Hogeveen 2005, Blowey y Edmondson 2010).

Entre los factores ligados al animal, se ha observado que el RCS es menor en vacas de primer parto y aumenta conforme se incrementa el número de parto (Mitev et al. 2012, McParland et al. 2013). Se han descrito también patrones irregulares de RCS a lo largo de la lactancia, con descensos rápidos al inicio y posteriores aumentos hacia el final de la lactancia (Rodríguez-Zas et al. 2000, de Haas et al. 2002, Atakan 2008).

Entre los factores raciales, Berry et al. (2007) reportaron una diferencia significativa entre el RCS de las vacas Holstein-Friesian $\left(\mathrm{RCS}=84965{\mathrm{CS} . \mathrm{ml}^{-1}}^{-1}\right.$ y Jersey $(\mathrm{RCS}=100709$ CS.ml- ${ }^{-1}$ ). Mediante la selección por bajos RCS en leche, es posible mejorar genéticamente el ganado lechero para reducir la incidencia de mastitis (Schutz 1994). La correlación genética entre la aparición de la mastitis clínica y RCS oscila entre 0,50 y 0,80 , con un promedio de 0,70 (Rupp y Boichard 2003), por lo que la selección para disminuir RCS debe aumentar la resistencia a la mastitis. A pesar de que la tasa de mejoría tiende 
a ser muy lenta, el costo económico que esto implica es menor en comparación con el costo de los tratamientos que se hacen contra la mastitis clínica y la pérdida en términos de producción de leche por casos de mastitis subclínica. Si bien el manejo es la manera más efectiva de corregir la calidad de la leche y los problemas de mastitis a corto plazo, el mejoramiento genético debe ser una parte importante de la estrategia para mejorar la calidad de la leche y la salud de la ubre a largo plazo (Shook 2001).

El rango reportado para la heredabilidad de RCS es muy amplio (Detilleux et al. 1997), con valores que oscilan desde 0,05 (Mrode y Swanson 1996) hasta 0,73 (Vallimont et al. 2009). Esta alta variación puede deberse, en parte, a los distintos modelos estadísticos y genéticos asumidos, los procedimientos de estimación y las poblaciones utilizadas en dichos estudios.

El presente estudio pretende estimar el efecto de factores genéticos aditivos y factores relacionados con la vaca lechera y el hato sobre el RCS durante la lactancia.

\section{MATERIALES Y MÉTODOS}

\section{Diseño y población}

Se realizó un estudio de carácter observacional y retrospectivo, mediante el análisis de la información recolectada en la base nacional de datos VAMPP (Noordhuizen y Buurman 1984, Pérez et al. 1989) administrada por la
Consultoría Regional en Informática para la Producción Animal Sostenible (CRIPAS), entre el 01/01/2002 y 31/08/2015.

Bajo este esquema, los productores lecheros ingresan voluntariamente la información de su animales (eventos productivos, reproductivos y sanitarios) al programa VAMPP Bovino. Esta información se envía periódicamente al programa CRIPAS, donde es sometida a revisión y edición para su análisis posterior. Los hatos participantes tienen diferentes periodos de seguimiento, que pueden variar desde pocos meses hasta más de 20 años. Asimismo, los periodos de seguimiento de las vacas y de las lactancias fueron de longitud variable.

Para la medición de los recuentos de células somáticas a partir de muestras individuales de leche de cada animal se utilizó el equipo Fossomatic $^{\mathrm{TM}}$ FC 200 (Foss 2015).

\section{Análisis estadístico}

Para el análisis estadístico, se transformó la variable original de conteo de células somáticas (RCS, en unidades por $\mathrm{ml}$ de leche) a un score en escala logarítmica (SCS), mediante la siguiente fórmula (Shook 1993):

$$
S C S=\log _{2}\left[\left(\frac{R C S}{100000}\right)+3\right]
$$

Esta variable fue analizada mediante un modelo animal mixto con repetibilidad, el cual se describe a continuación:

$$
Y_{i j k \text { lmnopqrs }}=\beta_{0}+\beta_{1} Z_{i}+\beta_{2} H_{j}\left(Z_{i}\right)+\beta_{3} R_{k}+\beta_{4} L_{l}+\beta_{5} M_{m}+\beta_{6} Y_{n}+\beta_{7} P_{o}+\beta_{8} K_{p}+A_{q}+A P_{r}+H S_{s}+\varepsilon_{i j k l n n o p q r s}
$$

donde:

$Y_{i j k l m n o p q}:$ SCS, Score de Células Somáticas.

- $\quad \beta_{0}$ : intercepto.

- $\quad \beta_{1} Z_{i}$ : efecto fijo para la i-ésima zona agroecológica (Clases: bosque húmedo montano/premontano, bosque muy húmedo tropical/premontano/montano y bosque pluvial montano); según la clasificación establecida por Holdridge (1987).

$\beta_{2} H_{j}\left(Z_{i}\right)$ : efecto fijo para la $j$-ésimo hato, anidado dentro de zona agroecológica (Clases: Hatos 1 hasta 237).

- $\quad \beta_{3} R_{k}$ : efecto fijo para la $k$-ésima raza (Clases: Holstein, Jersey, Holstein $\times$ Jersey). La clase Holstein $\times$ Jersey se conformó 
mayormente por animales reportados como $1 / 2 \mathrm{H}^{1 / 2} \mathrm{~J}$, y una minoría de animales con diferentes combinaciones de ambas razas.

- $\quad \beta_{4} L_{l}$ : efecto fijo para el l-ésimo número de lactancia (Clases: 1 hasta $>=6$ ).

$\beta_{5} M_{m}$ : efecto fijo para el $m$-ésimo mes del registro (Clases: Enero hasta Diciembre).

$\beta_{6} Y_{n}$ : efecto fijo para el $n$-ésimo año del registro (Clases: 2002 hasta el 2015).

$\beta_{7} P_{o}$ : efecto fijo para el $o$-ésimo periodo de lactancia (Clases: Periodos de 15,2 días desde 1 hasta $>=24$ ).

$\beta_{8} K_{p}$ : efecto fijo de para la p-ésima covariable de producción de leche $(\mathrm{kg})$ del día del registro.

$a_{q}$ : efecto aleatorio del q-ésimo animal, ligado a la genealogía. El archivo de genealogías estuvo constituido por un total de 36588 animales distribuidos en 18 generaciones. Los padres desconocidos fueron asignados a una sola población base.

$a p_{r}$ : efecto aleatorio del $r$-ésimo ambiente permanente. Cuantifica efectos ambientales que pueden influenciar el rendimiento de un animal de por vida.

- $\quad h s_{s}$ : efecto aleatorio de la r-ésima clase hato-semental. Cuantifica las posibles diferencias entre progenies de un mismo semental nacidas en distintos hatos.

$\varepsilon_{i j k l m n o p q r}$ : error aleatorio.

Este modelo se resolvió con el programa computacional ASREML (Gilmour et al. 1998) mediante el método de Máxima Verosimilitud Restringida REML (Harville 1977), obteniendo estimados de significancia estadística para efectos fijos y de magnitud de los componentes de varianza para efectos aleatorios.

Los estimados ajustados obtenidos a partir del modelo permitieron además identificar los grupos más propensos a contraer mastitis subclínica, es decir, aquellos que presentan tendencias a altos conteos de células somáticas.

A partir de los componentes de varianza se estimaron los parámetros genéticos de heredabilidad $\left(h^{2}\right)$ y repetibilidad $(r)$, mediante las siguientes fórmulas:

$$
\begin{gathered}
h^{2}=\frac{\sigma_{a}^{2}}{\sigma_{F}^{2}} \\
r^{2}=\frac{\sigma_{a}^{2}+\sigma_{a p}^{2}}{\sigma_{F}^{2}}
\end{gathered}
$$

donde:

$h^{2} \quad=$ índice de heredabilidad,

$r \quad=$ índice de repetibilidad,

$\sigma^{2}{ }_{\mathrm{a}} \quad=$ varianza genética aditiva,

$\sigma^{2}{ }_{\text {ap }}^{\mathrm{a}}=$ varianza atribuida a ambiente permanente,

$\sigma_{\mathrm{F}}^{2}=$ varianza Fenotípica.

Se obtuvieron además los estimados Mejores Estimadores Lineales Insesgados (BLUP, por sus siglas en inglés), los cuales en mejoramiento genético son interpretados como valores de cría para los individuos dentro de la población. Para cada BLUP, se calculó además un estimado de confiabilidad (o precisión) mediante la fórmula [4]:

$$
\operatorname{Conf}_{i}=\sqrt{\left(1-\frac{s_{i}^{2}}{\left(1+f_{i}\right) \sigma_{A}^{2}}\right)}
$$

donde:

$\operatorname{Conf}_{i}=$ valor de confiabilidad para el BLUP del $i$-ésimo individuo.

$\mathrm{s}_{i} \quad=$ error estándar reportado para el BLUP del $i$-ésimo individuo.

$\mathrm{F}_{i} \quad=$ coeficiente de consanguinidad del $i$-ésimo individuo.

$\sigma_{\mathrm{A}}^{2} \quad=$ varianza genética obtenida a partir de la matriz de parentescos (A).

\section{RESULTADOS Y DISCUSIÓN}

Se analizaron en total 198685 registros individuales de CS provenientes de 43535 lactancias (23 749 vacas) distribuidas en 237 hatos lecheros. La variable RCS presentó una 
distribución altamente sesgada (Promedio: 322

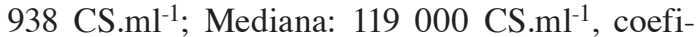
ciente de sesgo: 6,9), lo que se debe a una minoría de animales que presentan RCS muy elevados, superiores a 1 millón CS.ml ${ }^{-1}$. Por tal motivo, se utilizó la transformación logarítmica sugerida por Shook (1993) obteniéndose la variable SCS, que presentó una distribución aproximadamente normal (Promedio: 3,30; Mediana: 3,38; coeficiente de sesgo: 0,27). Este valor de SCS es equivalente a un RCS de $123114 \mathrm{CS}_{\mathrm{m}} \mathrm{ml}^{-1}$. El valor reconvertido (media geométrica) es el más utilizado como indicador de la incidencia de mastitis subclínica.

La solución al modelo estadístico propuesto (Cuadro 1) indicó un ajuste general altamente significativo $(\mathrm{p}<0,001)$. Al igual que todos los efectos evaluados fueron altamente significativos $(\mathrm{p}<0,001)$. Este resultado sin duda es influenciado por el elevado tamaño de la muestra, por lo que se hace necesario un análisis más detallado de cada factor.

Cuadro 1. Grados de libertad, valores F y valores de significancia (P) para los factores incluidos en el Análisis de Varianza de la variable Score de Células Somáticas (SCS).

\begin{tabular}{lccc}
\hline Fuente de variación & Grados de Libertad & Valor F & Valor P \\
\hline Ajuste general (Intercepto) & 1 & 52,2 & $<0,001$ \\
\hline Zona agroecológica & 5 & 12,1 & $<0,001$ \\
Hato (Zona) & 231 & 92,7 & $<0,001$ \\
Raza & 2 & 246,3 & $<0,001$ \\
Número de lactancia & 5 & 3042,7 & $<0,001$ \\
Mes & 11 & 14,7 & $<0,001$ \\
Año & 13 & 156,9 & $<0,001$ \\
Periodo de lactancia & 23 & 149,4 & $<0,001$ \\
Producción de leche & 1 & 5581,5 & $<0,001$ \\
\hline
\end{tabular}

En cuanto al efecto de zona se observó un valor significativamente menor de SCS para la zona bh-p y mayor para la zona bmh-p, en comparación con las demás zonas (Figura 1). Estas diferencias, aunque significativas, fueron de baja

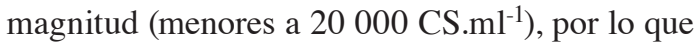
no sería prudente atribuirlas a factores climáticos. De mayor importancia son las diferencias significativas detectadas entre hatos (Figura 2), ya que en algunos casos son mayores a 4 puntos en SCS, lo que significa que el RCS de los hatos con conteos más altos es hasta 5 veces mayor que el de hatos con conteos más bajos. Esta variación entre los hatos puede deberse a múltiples razones, tales como sistema de manejo, confinamiento, métodos de control de mastitis, etc. Estos factores no fueron cuantificados en el presente estudio. Diversos estudios han revelado cómo el manejo y los procedimientos pre y post ordeño inciden sobre los RCS (Lévesque 2004, Blowey y Edmondson 2010, Ruegg y Pantoja 2013). Aspectos básicos de la rutina de ordeño, como lo son el presellado, el secado, los tiempos de ordeño y el sellado, son claves para mejorar significativamente los RCS (Ruegg y Pantoja 2013). El espacio en el área de confinamiento y materiales de las camas también son factores que inciden en los RCS (Lévesque 2004, Blowey y Edmondson 2010). 


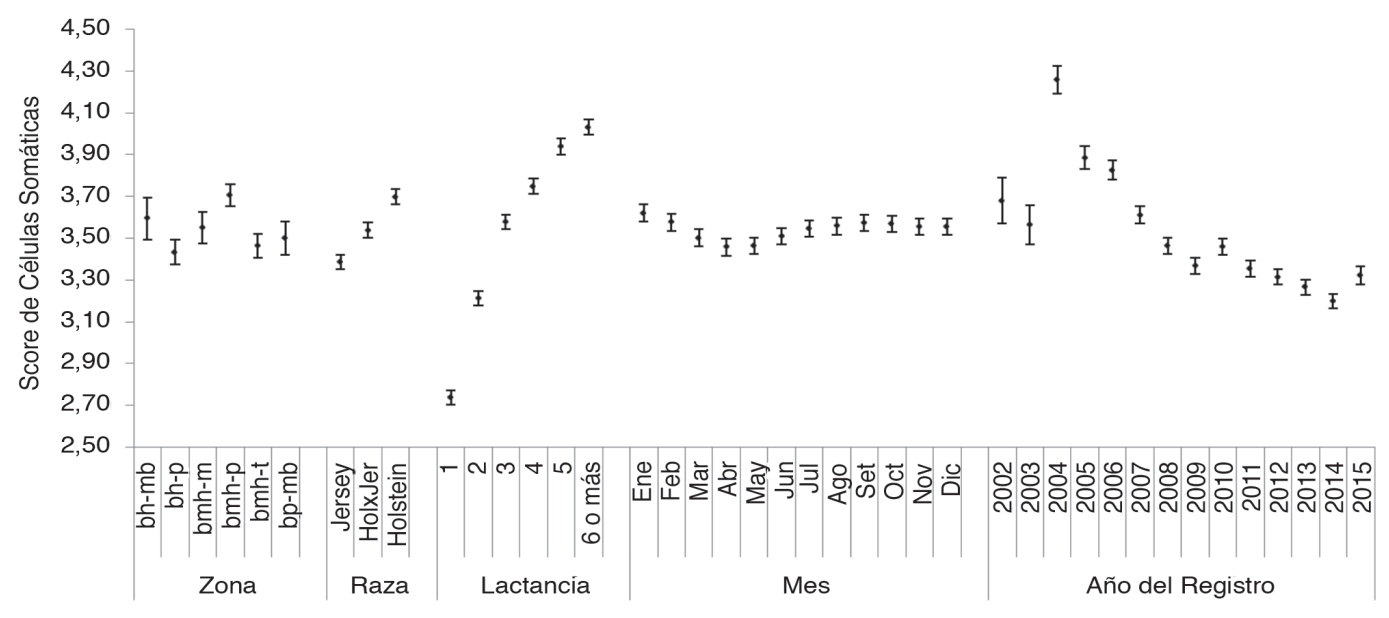

Fig. 1. Valores predichos e Intervalos de confianza 95\% de la variable Score de Células Somáticas (SCS) para todas las clases de los factores Zona, Raza, Lactancia, Mes y Año del Registro.

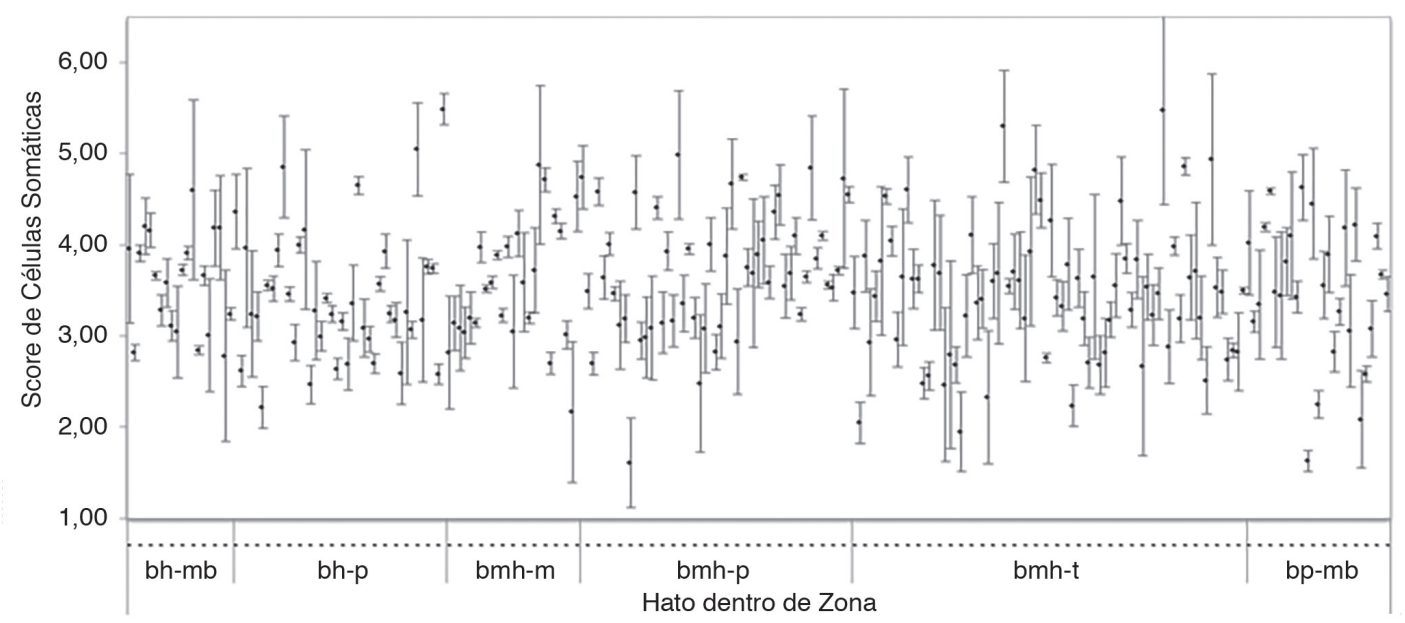

Fig. 2. Valores predichos e Intervalos de confianza 95\% de la variable Score de Células Somáticas (SCS) para hatos dentro de cada zona agroecológica (bh: bosque húmedo, bmh: bosque muy húmedo, bp: bosque pluvial, t: tropical, $\mathrm{m}=$ montano, $\mathrm{mb}$ : montano bajo, $\mathrm{m}$ : montano.

En cuanto a la raza, se observó un mayor SCS para las vacas Holstein $(\mathrm{SCS}=3,70)$ en comparación con el grupo Holstein $\times$ Jersey $(3,54)$ y Jersey $(\mathrm{SCS}=3,39)$. Estas diferencias, aunque significativas, fueron de baja magnitud. La casi linearidad entre los 3 estimados sugiere además que las diferencias raciales son principalmente aditivas. A diferencia del presente estudio, Berry et al. (2007) reportaron un mayor valor de SCS (log natural de RCS) para vacas Jersey $(\mathrm{SCS}=11,52 ; \mathrm{RCS}=100709$ células.ml-1), en comparación con vacas Holstein-Friesian ( $\mathrm{SCS}=11,35$;

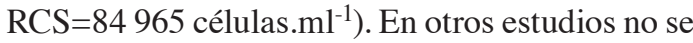
encontraron diferencias significativas para SCS 
entre las razas comparadas (Sewalem et al. 2006, Prendiville et al. 2010). Prendiville et al. (2010) obtuvieron promedios de SCS de 10,6; 10,8 y 10,7 para las razas Holstein, Jersey y Hosltein $\times$ Jersey. En vista de lo anterior, las diferencias observadas a nivel local entre estos grupos raciales no pueden ser generalizadas.

En cuanto al número de lactancia (Figura 1), se observó un patrón no lineal de aumento en SCS conforme estas avanzan, aspecto más destacado entre las lactancias 1 a 3. El valor obtenido para lactancias $\geq 6$ equivale a un RCS que duplica al obtenido para la primera lactancia. Este resultado es congruente con la tendencia descrita por McParland et al. (2013). Esta tendencia se asocia generalmente a una mayor exposición a contagio conforme aumenta el número de partos, debido a una mayor interacción entre animales sanos y enfermos (Ruegg y Pantoja 2013). Además puede relacionarse al desarrollo de casos crónicos en vacas que no son tratadas al momento de la aparición de la mastitis subclínica, o que no reciben una adecuada terapia de secado (Neijenhuis et al. 2001, McParland et al. 2013). Por otra parte la gran diferencia observada entre lactancias 1 y 2 puede relacionarse además con el hecho de que las vacas primerizas presentan mejores tasas de curación.

En el caso de los meses del registro (Figura 1) se observa que las vacas presentan mayores RCS en enero, mientras que en abril y mayo es cuando se presentan los menores RCS. No obstante, estas diferencias son de baja magnitud

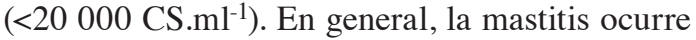
con mayor frecuencia en épocas con mayor calor y humedad, aunadas a un mayor estrés calórico y menor respuesta inmune por parte del animal (Ruegg y Pantoja 2013). En el presente estudio, aunque las diferencias son pequeñas, pueden deberse a que las vacas paridas en diciembre, enero y febrero son secadas en invierno, por lo que existe una mayor posibilidad de que se sequen enfermas, debido a la alta humedad y a las pocas o nulas rutinas correctas de secado. Después de 60 días de incubación, las vacas estarían por parir en verano, con mayores RCS. Por otro lado, durante abril y mayo la humedad ambiental es baja, por lo que la posibilidad de contaminación por bacterias ambientales es menor.

En cuanto al año del registro (Figura 1), se observa un comportamiento poco consistente entre el 2002 y 2004, lo cual puede ligarse a una menor disponibilidad de información para estos años. Posteriormente, a partir del 2004, se observa una tendencia consistente a la reducción en SCS a través de los años, que fue más evidente entre $2004(4,3)$ y $2009(3,4)$. Los años con menores RCS fueron del 2012 al $2015(3,3)$. Esta mejora a partir del 2004 puede ser debida a un aumento en la rigurosidad de las políticas de control por parte de la industria láctea (Vargas Bejarano 2008), la cual obliga a los productores a realizar mejoras en el manejo de los animales antes, durante y después de los ordeños, a tener una selección más rigurosa en relación con eventos de salud como la mastitis, y al desvío de la leche proveniente de animales con altos RCS. También se ha implementado un mayor seguimiento y asesoría en los programas de control de mastitis en las fincas.

En cuanto al efecto del periodo dentro de la lactancia (Figura 3), se observa una tendencia no lineal en SCS, que inicia con valores altos en el periodo 1 (1-15 días posparto, $\mathrm{SCS}=3,7$ ) para luego sufrir una reducción marcada hasta el periodo 4 (días 45 a 60 posparto, $\mathrm{SCS}=3,1$ ) donde se alcanza el mínimo de SCS. Posteriormente los valores aumentan progresivamente hasta alcanzar un máximo en el periodo 24 (días 350-365 posparto, $\mathrm{SCS}=3,9$ ). 


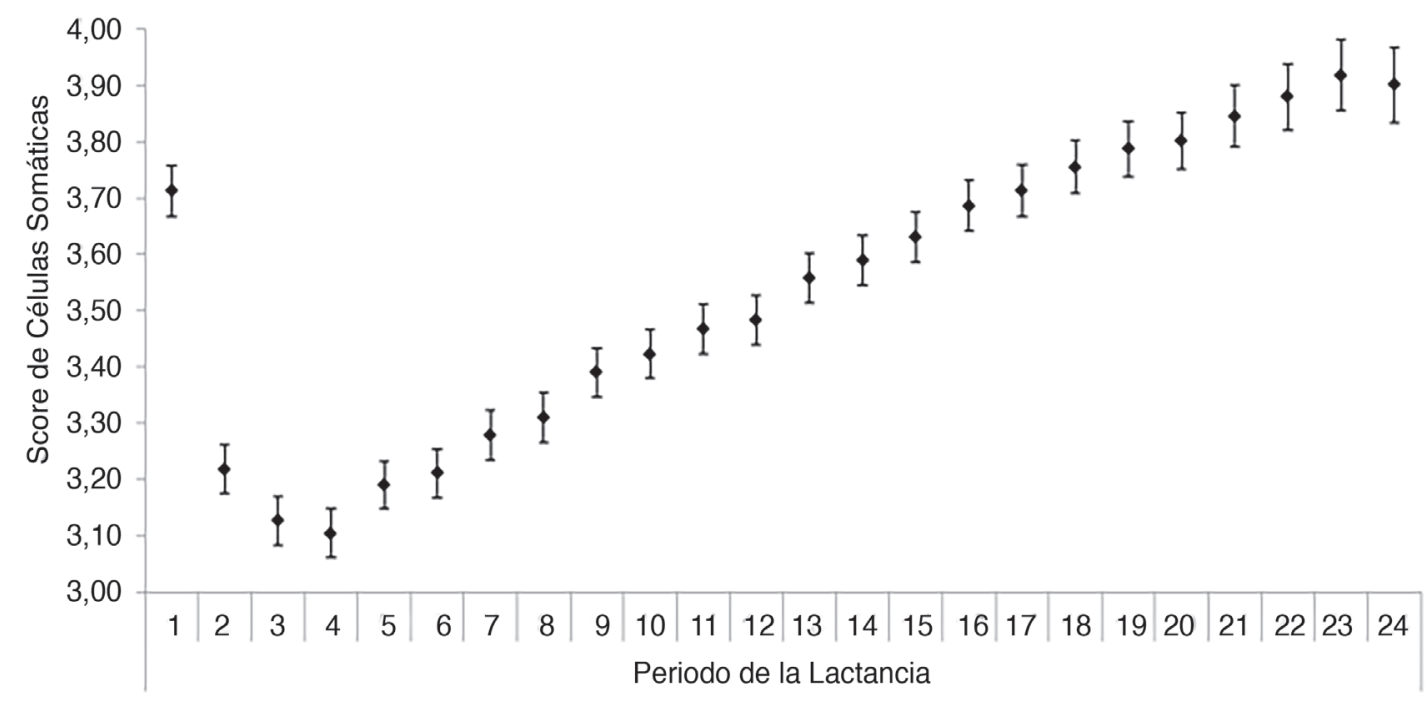

Fig. 3. Valores predichos e Intervalos de confianza 95\% de la variable Score de Células Somáticas (SCS) según periodo de la lactancia (1: 0-15 d posparto hasta 24:350-365 d posparto).

El comportamiento obtenido es similar al que describen Rodríguez-Zas et al. (2000), quienes mencionan que durante la lactancia el SCS hace un rápido descenso al inicio, con un subsecuente aumento después del décimo día posparto, y una estabilización o leve descenso al final de la lactancia. De Haas et al. (2002) y Atakan et al. (2008) también encontraron un efecto significativo del periodo de lactancia sobre el SCS. Los autores observaron que después del segundo mes de lactancia, los RCS descienden, aspecto que muestra los valores más bajos durante el segundo y tercer mes de lactancia. Posteriormente, al cuarto mes, estos conteos empiezan a incrementarse hasta el final de la lactancia.

El patrón observado podría asociarse con un efecto normal de menor/mayor concentración de CS en los estadíos de mayor/menor producción de leche. Sin embargo, esta no es una explicación válida para el presente estudio, ya que la producción al día de registro fue considerada como covariable dentro del modelo. Otros autores indican que el patrón observado, aunque frecuente, no es normal en el caso de vacas sanas
(Ruegg y Pantoja 2013). Estos autores proponen que el valor alto de SCS al inicio de la lactancia se asocia mayormente con infecciones oportunistas en el posparto causadas principalmente por patógenos ambientales, mientras que el incremento observado al final de la lactancia se relaciona con una mayor exposición a patógenos contagiosos conforme progresa la lactancia, debido a una mayor interacción animal sano y animal enfermo. Además, este aumento en la proliferación de bacterias se puede ver favorecido por una mayor manipulación de la ubre y una mayor abertura del pezón conforme avanzan los días, lo que se vería reflejado en un aumento de los RCS.

En relación con la covariable de producción, se observó una relación inversa (favorable) entre nivel de producción y SCS, con una reducción de 0,051 en SCS por cada incremento de $1 \mathrm{~kg}$ en producción diaria de leche. Cabe agregar que la producción promedio diaria en la población analizada fue de $21,3 \mathrm{~kg}(\mathrm{DE}=7,9)$ y la correlación fenotípica entre ambos rasgos fue de $-0,18(\mathrm{p}<0,001)$. Este es un resultado muy similar al reportado por Al Seaf et al. (2007) quienes 
hallaron una correlación fenotípica de - 0,15 entre ambos rasgos. Carlén et al. (2004) reportaron correlaciones ambientales de $-0,22,-0,15$ y $-0,13$ entre SCS y producción de leche en primera, segunda y tercera lactancia, respectivamente. En nuestro estudio, la correlación negativa puede estar ligada a factores ambientales, ya que las vacas con mayores producciones podrían estar en hatos que a su vez tienen un mejor control de mastitis. Cabe recalcar, sin embargo, que a nivel genético existen resultados consistentes en la literatura que indican una asociación positiva (desfavorable) entre SCS y rasgos de producción (Mrode y Swanson 1996, Carlén et al. 2004). En el presente estudio no se calcularon correlaciones a nivel genético entre ambos rasgos.

En cuanto a los factores aleatorios, el Cuadro 2 resume los componentes de varianza obtenidos a partir de la solución del modelo [1] y los estimados de heredabilidad y repetibilidad derivados a partir de estos componentes. Como se observa, el índice de heredabilidad $\left(h^{2}\right)$ obtenido fue de 0,08 , mientras que la repetibilidad $(r)$ fue de 0,33. Mrode y Swanson (1996) obtuvieron valores de $h^{2}$ entre 0,05 y 0,47 . De igual manera Haile-Mariam et al. (2001) reportaron valores de $h^{2}$ entre 0,04 y 0,05 al principio, y de 0,11 a 0,13 al final de la primer lactancia; mientras que en la segunda y tercer lactancia encontraron valores de 0,09 a 0,11. Carlén et al. (2004) también reportaron un resultado similar de $h^{2}$, con valores entre $0,10 \mathrm{y}$ 0,14 . Costa et al. (2010), en ganado Holstein brasileño, obtuvieron valores de 0,07 y 0,34 para $h^{2}$ y $r$, respectivamente, muy similares a los encontrados en el presente estudio. De igual manera Zhao et al. (2015) encontraron valores de 0,05 y 0,20 para $h^{2}$ y $r$, respectivamente; con ligeros incrementos en $h^{2}$ en relación con el número de partos.

Cuadro 2. Componentes de varianza para Score de Células Somáticas SCS obtenidos a partir de la solución del modelo [1].

\begin{tabular}{lr}
\hline Componente & Valor \\
\hline Varianza residual $(r)$ & 1,884 \\
Animal $(a)$ & 0,240 \\
Ambiente permanente $(a p)$ & 0,720 \\
Interacción Hato-Semental $(h s)$ & 0,078 \\
Varianza Fenotípica $(F=r+a+a p+h s)$ & 2,922 \\
Heredabilidad $(a / F)$ & $0,08(\mathrm{DE}=0,008)$ \\
Repetibilidad $(a+a p) / F$ & $0,33(\mathrm{DE}=0,004)$ \\
\hline
\end{tabular}

El estimado de $h^{2}$ obtenido en el presente estudio ratifica que el componente genético juega un papel importante en el valor de SCS, similar al reportado para otras latitudes. Cabe señalar que esta variable es considerada desde hace varios años en programas de mejoramiento genético para ganado lechero (Schutz 1994). Entre los 10 países que participan en INTERBULL, 6 incluyeron en sus índices totales de mérito el rasgo de mastitis, el cual considera tanto el SCS como el índice de mastitis (INTERBULL 2015).
De manera similar, la CDN (Canadian Dairy Network) inició recientemente evaluaciones genéticas para resistencia a mastitis en las razas Holstein, Ayrshire y Jersey (Jamrozik et al. 2013, Miglior et al. 2014, CDN 2014).

En cuanto a los BLUP, la gran mayoría de los animales se incluyeron en el rango entre -1 y 1 puntos SCS con respecto al promedio, lo que ratifica una importante variabilidad genética para SCS dentro de la población analizada. La confiabilidad para los BLUP, obtenida mediante 
la fórmula [4], presentó un valor promedio de $0,40(\mathrm{DE}=0,16)$ en la población total. En las vacas con registros de SCS la confiabilidad promedio fue de 0,43 ( $\mathrm{DE}=0,14)$, y varió principalmente en función del número de registros de SCS y lactancias disponibles por vaca. Para las vacas sin registros de SCS la confiabilidad promedio fue de 0,24 ( $\mathrm{DE}=0,15)$, con variación principalmente, en función del número de hijas con datos de SCS. En cuanto a los toros se obtuvo un valor promedio de confiabilidad de 0,44 $(\mathrm{DE}=0,20)$ con variación también en función del número de hijas con datos de SCS.

En general, los resultados obtenidos son similares a otros estudios. Los efectos climáticos, aunque significativos, no son de gran magnitud. Por el contrario, existen amplias diferencias entre hatos, por lo que es necesario ahondar más en las razones ligadas a estas grandes diferencias.

Los resultados obtenidos permitieron identificar los grupos dentro de la población que tienden a presentar mayores conteos de células somáticas y que por tanto tienen mayor propensión a contraer mastitis subclínica. En este sentido, las medidas preventivas deben reforzarse principalmente en vacas de edad avanzada y en etapas de lactancia tempranas o tardías. Debido a que gran parte de los casos de mastitis se deben a infecciones intramamarias ocurridas en el periodo seco o que persistieron a lo largo de este periodo, es importante implementar terapias de secado adecuadas, principalmente en las épocas críticas. Una adecuada terapia de secado debe iniciar por una disminución progresiva de la energía en la dieta, con el fin de reducir la producción antes del secado, seguida del uso de un antibiótico de secado de larga acción debidamente aprobado y, en caso de alta exposición a patógenos, utilizar además un sellador interno o externo en las 4 tetas (NMC 2016).

Se observan amplias diferencias en RCS a nivel individual. Esto ratifica la importancia de llevar registros individuales de RCS y realizar muestreos periódicos (p.e mensuales) a lo largo de lactancia. Esta información permite realizar un mejor control de la salud de la ubre e implementar manejos diferenciados, a la vez que sirve como un indicador de la susceptibilidad de cada vaca y provee un insumo indispensable para el cálculo de valores genéticos. Importante identificar y descartar aquellas vacas con RCS altos y repetidos, también evitar el uso de toros con evaluaciones genéticas desfavorables para SCS.

La inclusión del SCS en los índices de selección puede disminuir la susceptibilidad a esta enfermedad en el mediano plazo; además de mejorar la eficiencia económica de las fincas lecheras.

\section{LITERATURA CITADA}

Al-Seaf, A; Keown, JF; Van Vleck, LD. 2007. Estimates of correlations among yield traits and somatic cell score with different models to adjust for bovine somatotropin effects on Holstein dairy cows. Genetics and Molecular Research 6(1):67-78.

Atakan, KOÇ. 2008. A Study of Somatic Cell Counts in the Milk of Holstein-Friesian Cows Managed in Mediterranean Climatic Conditions. Turkish Journal Veterinary Animal Science 32(1):13-18.

Berry, DP; Lee, JM; Macdonald, KA; Stafford, K; Matthews, L; Roche, JR. 2007. Associations among body condition score, body weight, somatic cell count, and clinical mastitis in seasonally calving dairy cattle. J. Dairy Sci. 90(2):637-648.

Blowey, RW; Edmondson, P. 2010. Mastitis Control in Dairy Herds. 2 ed. CAB International, Oxfordshire, UK. 266 p.

Carlén, E; Strandberg, E; Roth, A. 2004. Genetic Parameters for Clinical Mastitis, Somatic Cell Score, and Production in the First Three Lactations of Swedish Holstein Cows. J. Dairy Sci. 87(9):3062-3070.

CDN (Canadian Dairy Network,CA). 2014.Mastitis Resistance Selection: Now a Reality! (en línea). Guelph, Canada. Consultado 11 set. 2015. Disponible en https://www. google.com/url?sa=t\&rct=j\&q=\&esrc=s\&source $=$ web\&cd=1\&ved=0CCIQFjAAahUKEwiGwfe7nO_ HAhUGjg0KHWxsCow\&url=https $\% 3 \mathrm{~A} \% 2 \mathrm{~F} \% 2$ Fwww.cdn.ca\%2Fdocument.php\%3Fid\%3D356\& usg=AFQjCNHWzF1ZKBdDhBdrx1yj-uy0A2phA\&bvm=bv.102537793, d.eXY\&cad=rja

Costa, C; Freitas, A; Cobuci, J; Thompson, G; Carvalheira, J; Silva, M; Valloto, A. 2010. Genetic Parameters for Test Day Somatic Cell Count in Brazilian Holstein Cattle. Proceedings of the 9 th World Congress on Genetics Applied to Livestock Production. Leipzig, Alemania. p. 1-31. 
De Haas, Y; Barkema, HW; Veerkamp, RF. 2002. The Effect of Pathogen-Specific Clinical Mastitis on the Lactation Curve for Somatic Cell Count. J. Dairy Sci. 85(5):1314-1323.

Detilleux, J; Leroy, P; Volckaert, D. 1997. Alternative use of somatic cell counts in genetic selection for mastitis resistance. Proceedings of the international workshop on genetic improvement of functional traits in cattle health. Uppsala, Sweden. p. 34-44.

Foss. 2015. Fossomatic ${ }^{\mathrm{TM}}$ FC (en línea). Hilrode, Denmark. Consultado 28 may. 2015. Disponible en http://www. foss.dk/industry-solution/products/fossomatic-fc/

García, AD. 2004. Células somáticas y alto recuento bacteriano. ¿Cómo controlarlo? (en línea). Consultado 5 jul. 2011. South Dakota, USA. Disponible en http:// pubstorage.sdstate.edu/AgBio_Publications/articles/ ExEx4031-S.pdf

Gilmour, AR; Cullins, BR; Welham, SJ; Thompson, R. 1998. ASREML user's manual. New South Wales Agriculture, Orange Agricultural Institute, Orange, NSW, Australia. p. 104

Haile-Mariam, M; Goddard, ME; Bowman, PJ. 2001. Estimates of Genetic Parameters for Daily Somatic Cell Count of Australian Dairy Cattle. J. Dairy Sci. 84(5):1255-1264.

Harmon, RJ. 2001. Somatic cell counts: a primer. Reno, Nevada, Estados Unidos. 40th Annual Meeting, National Mastitis Council. 6 p.

Harville, DA. 1977. Maximum Likelihood Approaches to Variance Component Estimation and to Related Problems. J. Am. Stat. Assoc. 72(358):320-338.

Hogeveen, H. 2005. Mastitis in Dairy Production: Current Knowledge and Future Solutions. The Netherlands, Wageningen Academic Pub. 744 p.

Holdridge, L. 1987. Ecología basada en zonas de vida. Traducido por Humberto Jiménez Saa. San José, Costa Rica. IICA. 216 p.

INTERBULL (International Bull Evaluation Service, SE). 2015. International Bull Evaluation Service Official Website (en línea). Uppsala, Sweden. Consultado 9 set. 2015. Disponible en http://www.interbull.org/ index

Jamrozik, J; Koeck, A; Miglior, F; Kistemaker, GJ; Schenkel, FS; Kelton, DF; Van Doormaal, BJ. 2013. Genetic and Genomic Evaluation of Mastitis Resistance in Canada. Nantes, France. INTERBULL Bulletin (47):43-51.

Lévesque, P. 2004. Menos mastitis, mejor leche. Québec, Canada, Institut de Technologie Agroalimetaire. 96 p.

McParland, S; O’Brien, B; McCarthy, J. 2013. The association between herd- and cow- level factors and somatic cell count of Irish dairy cows. Irish J. Agr. Food Res. 52(2):151-158

Miglior, F; Koeck, A; Jamrozik, J; Schenkel, FS; Kelton, DF; Kistemaker, GJ; Van Doormaal, BJ. 2014. Index for Mastitis Resistance and Use of BHBA for Evaluation of Health Traits in Canadian Holsteins. Berlin, Germany. INTERBULL Bulletin (48):73-78.

Mitev, JE; Gergovska, ZHI; Miteva, TCHM. 2012. Effect of teat end hyperkeratosis on milk somatic cell counts in Bulgarian Black-and-White dairy cattle. Bulgarian J. Agric. Sci. 18(3):451-454.

Mrode, RA; Swanson, GJT. 1996. Genetic and statistical properties of somatic cell count and its suitability as an indirect means of reducing the incidence of mastitis in dairy cattle. Anim. Breed. Abs. 64:847-857.

Neijenhuis, F; Mein, GA; Britt, JS; Reinemann, DJ; Hillerton, JE; Farnsworth, R; Baines, JR; Hemling, T; Ohnstad, I; Cook, N; Morgan, WF; Timms, L. 2001. Evaluation of bovine teat condition in commercial dairy herds: 4. Relationship between teat-end callosity or hyperkeratosis and mastitis. (disco compacto) National Mastitis Council. 2th International Symposium on Mastitis and Milk Quality. Vancouver, BC, Canada. 1 disco compacto, $8 \mathrm{~mm}$.

NMC (National Mastitis Council, USA). 2016. Recommended mastitis control program-International version (en línea). Wisconsin, USA. Consultado 30 ago. 2016. Disponible en https://www.nmconline.org/docs/ NMCchecklistInt.pdf

Noordhuizen, JPTM; Buurman, J. 1984. Veterinary automated management and production control program for dairy farms (VAMPP). The application of MUMPS for data processing. Vet. Q. 6(2):66-72.

Pérez, E; Baayen, MT; Capella, E; Barkema, H. 1989. Development of a livestock information system for Costa Rica. In Kuil, H; Palin, RW; Huhn. JE (eds.) Livestock Production and Diseases in the Tropics. Proc. IV Internl. Conf. Inst. Trop. Vet. Med. Utrecht, The Netherlands. p. 221-224.

Prendiville, R; Pierce, KM; Buckley, F. 2010. A comparison between Holstein-Friesian and Jersey dairy cows and their F1 cross with regard to milk yield, somatic cell score, mastitis, and milking characteristics under grazing conditions. J. Dairy Sci. 93(6):2741-2750.

Rodriguez-Zas, SL; Gianola, D; Shook, GE. 2000. An Approximate Bayesian Analysis of Somatic Cell Score Curves in Holsteins. Acta Agric. Scand., Sect. A, Animal Sci. 50(4):291-299.

Ruegg, PL; Pantoja, JCF. 2013. Understanding and using somatic cell counts to improve milk quality. Irish J. Agric. Food Res. 52(2):101-117.

Rupp, R; Boicchard, D. 2003. Genetics of resistance to mastitis in dairy cattle. Vet. Res. 34(5):671-688.

Schutz, MM. 1994. Genetic Evaluation of Somatic Cell Scores for United States Dairy Cattle. J Dairy Sci. 77(7):2113-2129.

Sewalem, A; Miglior, F; Kistemaker, GJ; Van Doormaal, BJ. 2006. Analysis of the Relationship Between Somatic Cell Score and Functional Longevity in Canadian Dairy Cattle. J. Dairy Sci. 89(9):3609-3614. 
Sharma, N; Singh, NK; Bhadwal, MS. 2011. Relationship of Somatic Cell Count and Mastitis: An Overview. Asian-Aust. J. Anim. Sci. 24(3):429-438.

Shook, G. 2001. Breeding, selection and somatic cell counts: Where are we today? (en línea). Wisconsin, USA. Consultado 15 mar. 2015. Disponible en http://www. nmconline.org/articles/GenSCS.pdf

Shook, GE. 1993. Genetic improvement of mastitis through selection on somatic cell count. Vet. Clin. North Am. Food Anim. Pract. 9(3):563-581.

USDA (Department of Agriculture, USA). 2007. Dairy 2007 Part I: Reference of Dairy Cattle Health and Management Practices in the United States, 2007 (en línea). Colorado, USA. Consultado 15 mar. 2015. Disponible en http://www.aphis.usda. gov/animal_health/nahms/dairy/downloads/dairy07/ Dairy07_dr_PartI.pdf

Vallimont, JE; Dechow, CD; Sattler, CG; Clay, JS. 2009. Heritability estimates associated with alternative definitions of mastitis and correlations with somatic cell score and yield. J. Dairy Sci. 92(7):3402-3410.

Vargas Bejarano, R. 2008. La calidad de la leche en Costa Rica. La Cooperativa de Productores de Leche Dos Pinos: Un caso de éxito. In Barbosa, SBP; Batista, AMV; Monardes, H (eds.). Congresso Brasileiro de Qualidade do Leite. CCS Gráfica e Editora (3, 2008, Recife, Brasil). Recife, Brasil. p. 241-250.

Zhao, F; Guo, G; Wang, Y; Guo, X; Zhang, Y; Du, L. 2015. Genetic parameters for somatic cell score and production traits in the first three lactations of Chinese Holstein cow. J. Integr. Agric. 14(1):125-130. 